\title{
Nursing Assessment of Pain during Spontaneous Perineal Tear Repairing by Comparing Two Pharmacological Methods
}

\author{
Aya Mostafa Mohamed ${ }^{1}$, Hamida Alam Eldin ${ }^{2} \&$ Ahmed Mohamed Abbas ${ }^{3}$. \\ ${ }^{1}$ Clinical Demonstrator of Obstetrics \& Gynecological Nursing, Faculty of Nursing, Assiut University \\ ${ }^{2}$ Assistant Professor of Obstetrics \& Gynecological Nursing, Faculty of Nursing, Assiut University \\ ${ }^{3}$ Lecturer of Obstetrics \& Gynecology, Faculty of medicine, Assiut University
}

\begin{abstract}
Background: pain is a nursing diagnosis and its assessment and management are nurses' primary responsibility. This study aimed to: assess perineal pain after topical application of lidocaine-prilocaine cream and lidocaine infiltration during repair of spontaneous postnatal perineal tears. Patients and methods: this study was conducted at the reception unit at Women's Health Hospital, Assiut University in the period from October 2016 to March 2017. The study sample included 144 women with spontaneous postnatal perineal tear divided in to two groups (72 women in each group); the lidocaine/prilocaine (LP) cream group and the lidocaine infiltration group. The pain score was measured in each group during suturing using the visual analogue scale. Results: the mean pain score was $3.86 \pm 1.59$ in LP group and $5.99 \pm 1.47$ for lidocaine group. $76.4 \%$ of women in LP group and $30.6 \%$ in lidocaine group were satisfied. Conclusions: the findings of this trial concluded that pain is better when using LP cream than using lidocaine infiltration during repair of postnatal spontaneous perineal tears. Recommendations: as the pain is the most common problem in the postnatal period; the study emphasized the importance of postnatal care and good pain management by the nurse.
\end{abstract}

\section{Keywords: Perineal Tear; Pain; Lidocaine Prilocaine (Lp)\& Lidocaine Infiltration.}

\section{Introduction}

One of the most commonly reported maternal health problems after birth is perineal pain associated with perineal trauma; it is estimated that approximately $70 \%$ of women who have a vaginal birth will sustain perineal trauma requiring suturing. No doubt, for some women, the experience of perineal pain and trauma can impact their recovery from birth (Bick et al., 2013).

Perineal trauma at birth is complains with perineal pain, dyspareunia, perineal infection, fistula, and fecal incontinence (Manzanares et al., 2013). Prevention of perineal trauma is one of the best options for birthing women. So both child-bearing women and health professionals place a high value for minimizing perineal trauma and reducing potential associated morbidity for mothers (Dönmez \& Kavlak, 2015).

Pain perception is a complex culmination of physiologic activities that begins with sensory nerve stimulation. The neuromatrix theory of pain reminds us that pain perception is a whole mind-body-spirit phenomenon, with previous experiences coloring a woman's view at any given moment. How a woman feels about her birth experience may also affect her perception of pain in the postpartum period. Women who had been able to make a conscious, informed choice about how to best cope with the pain of their labor and maintain some sense of control often reported greater satisfaction with their birth experiences. Likewise, when a woman senses that her emotional needs have been met, a sense of coping and accomplishment often prevails and vice versa (Eshkevari et al., 2013).

Several nursing interventions have been introduced for reducing risk of childbirth related perineal trauma, such as antenatal perineal massage, controlling \& slowing the expulsion of baby's head combined with manual perineal protection, coaching the mother during pushing, episiotomy, using perineal warm compresses during second stage of labour, stretching perineum during labour, using oils or lubricants \& use of different pushing techniques (Laine et al., 2016).

Removing pain and factors which interrupt a woman's natural enjoyment of the early postnatal period is vital in the promotion of bonding, breastfeeding and overall improved women and child health (Moore \& Moorhead, 2013).

Pain is a nursing diagnosis and its assessment and management are nurses' primary responsibility as the nurse is often the healthcare professional most involved in on-going assessment, implementing the approved pain management intervention and evaluating the response to such interventions. Health care professionals must be knowledgeable about effective and compassionate pain relief medications (Oregon State Board of Nursing, 2016).

Pain assessment and management serves to improve the quality of life for those patients who suffer from pain as well as to reduce the morbidity and costs associated with untreated or inappropriately treated 
pain. Pain is one of the most common reasons clients seek medical attention, and are a symptom that is encountered by every health care provider. An individual's self-report of pain, along with level of functional impairment are the optimal standards upon which pain management interventions are based. In the absence of ability to self -report level of pain, an appropriate non-verbal scale should be used. The management of pain must be a priority for nurses and all others who provide care to individuals in pain (Oregon State Board of Nursing, 2016).

Midwives' roles during repair are: explain to the women what they plan to do and why, offer analgesia, ensure good lighting, position the women and document the assessment \& its results. She should develop the trust with the women to make the experience less traumatic. The permanent presence of midwives in perineal repair minimizes the problems associated with the rotation of inexperienced junior medical staff. There is also evidence to suggest that women prefer to be sutured by midwives (The Royal College of Midwives, 2012).

\section{Significance of the study}

Following vaginal birth, women commonly reported pain from perineal trauma. This pain affects women's ability to mobilize (East et al., 2012). Studies conducted in obstetrical operations are more focused on pain during second stage of labor or pain in the early postpartum period. Few studies have focused on pain during perineal repair (Kargar et al., 2016).

\section{Aim of the study}

This study aimed to assess perineal pain level and overall mother's satisfaction by comparing the analgesic effect of topical application of lidocaineprilocaine cream and lidocaine infiltration during repair of postnatal spontaneous perineal tears.

\section{Patients \& Method \\ Technical design \\ Research design}

The current study used a randomized open-labeled trial.

\section{Setting of the study}

This study was conducted at the reception unit at Women's Health Hospital, Assiut University

\section{Patients}

The study enrolled all women who were delivered at the reception unit at Women's Health Hospital, Assiut University when they had spontaneous perineal tear after vaginal birth and met the inclusion criteria during the study period. Patients divided into two groups; group (1) received lidocaine infiltration and group (2) received lidocaine prilocaine (LP) cream.

\section{Study Outcomes \\ Primary Outcome}

Mean pain score during suture of spontaneous perineal tears

\section{Secondary Outcomes}

Pain by degree of perineal tear, overall women' satisfaction after the procedure, need for additional anesthetics, side effects of both used anaesthetics drugs and duration of perineal repair.

\section{Inclusion criteria}

The inclusion criteria included multiparous and primiparous women who admitted for normal vaginal delivery with spontaneous perineal tear, women who accepted participation in the study, gestational age 37 weeks and more and uncomplicated pregnancy.

\section{Exclusion criteria}

Women were excluded because of sensitivity to topical or local anesthetics, medical and obstetrical problems such as liver, kidney, uncontrolled diabetes and heart diseases, using forceps for delivery, presence of cervical tears and presence of several types of tears.

\section{Tools of the study}

Two tools were used for data collection. It was designed and utilized by the researchers to collect the required data for every selected woman at the selected setting.

Tool (1):Structured interview schedule

Part 1: personal data of participating women which included name, age, residence, educational level, sensitivity and medical history.

Part 2: current pregnancy and labour outcomes which included parity, gestational age, complications during pregnancy, birth weight in gm., cranial circumference $(\mathrm{cm})$ and degree of perineal laceration (first, second, third or fourth degree).

Part 3: overall women's satisfaction with the used anesthetic agents using a Likert scale question with three options (satisfied, satisfied to some extent, dissatisfied).

Part 4: included the following: need for additional analgesia, duration of perineal repair and side effects of anaesthetics used.

Tool (2): (Visual Analog Scale (VAS)) Pain scale It was used for assessment of women's preception toward pain. The scale is named after its inventor, psychologist (Rensis, 1932). It is a measuring instrument for subjective characteristics that cannot be directly measured. It was ranged from $0-10 \mathrm{~cm}$ where 0 represents no pain, score $(1,2,3)$ represent mild pain, $(4,5,6)$ represent moderate pain and $(7,8$, $9 \& 10)$ represent severe pain. 


\section{Operational design \\ Preparatory phase}

The researcher reviewed related literature (local \& international) using textbooks, web articles and scientific magazines then the tools were prepared. Validity

The tools were reviewed for validity by 3 experts in obstetrics and gynecology (medical \& nursing).

\section{Pilot study}

A pilot study was carried out after preparation \& revision of the study tools for validation by supervisors and before starting the data collection on $10 \% \quad(\mathrm{n}=14)$ women who were delivered vaginally and had spontaneous perineal tear to test the clarity and feasibility of the study tools. These women included in the study sample.

\section{Field work}

The study was carried out during the period from October 2016 to March 2017.

\section{Limitations of the study}

The main limitation of the study was the subjectivity in reporting pain through VAS scale, and reporting satisfaction through Likert scale as there are no objective parameters to assess pain and satisfaction. Refused participation in the study from some women was another limitation. Finally, most women had a dread of encountering pain much more terrible than they expected; believing that new treatment might be less effective than conventional one.

\section{Ethical considerations}

Oral consent was obtained from women who were participated in the study, after explaining the nature and purpose of the study. There was no any risk for the women during conduction of the study. The study was followed common ethical principles in clinical research. Confidentiality and anonymity would be assured and the participating women had the right to refuse participation or withdraw from the study without any rational.

\section{Administrative design}

The study was approved from ethical committee of the Faculty of Nursing; Assiut University and obtaining an official permission from the manager of Woman's Health Hospital to proceed with the study.

\section{The procedure}

Firstly the studied women were assessed by the investigator for

- Previous and current obstetrical history (parity, gestational age, pregnancy induced hypertension $(\mathrm{PIH})$ gestational diabetes, preeclampsia, eclampsia, anemia, polyhydrominos, oligohydromnios, placenta previa, placenta abruption and antepartum hemorrhage (APH).
- Any medical problems includes (uncontrolled diabetes mellitus, neurological diseases which affect lower limb, heart, kidney and liver diseases)

- Previous adverse reaction to topical and a local anesthetic.

- If the women were received any systemic analgesics for intra-partum pain control.

- Also women were assessed for presence of cervical tear, several types of rupture and bleeding.

- After excluding any woman from participation in the study, the investigator was explained the nature $\&$ purpose of the study and give the women full description and information needed about the study, then oral informed consent and basic demographic information was obtained.

- After that the participating women were randomized in a 1:1 ratio to either local injection of lidocaine (Group I); or application of LP cream (Group II) for pain relief during perineal repair. Randomization was done using a computer generated table of random numbers with allocation concealment. The allocation treatment had written on cards and was closed in sequentially numbered, opaque and stapled envelopes. The envelopes were opened after the enrolled participants completed all baseline assessments. After allocation to both groups, the women were asked to report any problem and additional anesthesia was requested during perineal repair whenever pain exceeded the tolerability threshold. Once allocation had been done, it could not be changed.

- After randomization; the women were began to receive the allocation treatment as the follow:

- Visual inspection of the perineum was done by the investigator to identify the laceration $\&$ its degree and location; also sources of bleeding were identified and controlled before repair if present.

- In the lidocaine injection group, the resident obstetrician injected $5 \mathrm{~mL}$ of $2 \%$ lidocaine HCL anhydrous solution slowly along the lines of the edges of the perineal tears after delivery, with frequent aspirations to avoid intravascular injection and the suture procedure was delayed for 5 minutes after the injection.

- In the topical lidocaine prilocaine (LP) group, the investigator applied 5-g dose of LP cream to the intact tissue around the tear for 5 minutes before suturing had occurred to have time to take effect. If there was active bleeding during this period, packing method was used.

- After that in all women, perineal tears were repaired with a loose, continuous, non-locking suture to close the vaginal mucosa and the muscular layer of the perineum; the skin was closed with the same continuous suture to approximate the subcutaneous 
tissue. The same suturing material, a rapidabsorption polyglactin 910 suture (2-0 Vicryl rapid) was used for repair.

- Women were placed in the lithotomy position for repair and suturing technique was done by resident obstetrician. The investigator was observed the women and assessing pain during repair. After allocation treatments were received $\&$ tears were sutured and before leaving the reception unit (approximately 2 hours after delivery) the investigator was interviewed the women for about 15 minutes for each woman and was completed recording of maternal demographics, delivery details, perineal trauma characteristics and other parts of the study tools. Moreover, the request of additional anesthetic $(5 \mathrm{~mL}$ of $2 \%$ lidocaine solution for both groups) during delivery was recorded. Assessment of the perineal wound and any adverse effects for the allocation treatments were done by the investigator and same antibiotic regimen was used for all women for prevention of wound infection. The investigator was asked each woman to record the severity of pain that she had experienced during perineal repair in a $10-\mathrm{cm}$ VAS pain scale where $0 \mathrm{~cm}$ means no pain and $10 \mathrm{~cm}$ means unbearable pain by asking the women to mark the point that best indicated the perception of her pain on the VAS scale. The level of pain was estimated in $\mathrm{cm}$ from the length of the space running from the left hand and the point that the women had marked and also the women were asked to express their overall satisfaction with the anesthesia method that used during perineal repair using a Likert scale question with three options (satisfied, satisfied to some extent, dissatisfied). Finally, health education was done by the investigator regarding perineal tear, postpartum perineal wound care and wound infection symptoms such as pain, suppuration, fever, vulval edematous, redness and swollen mucosa was described for women. And they were advised to refer these symptoms to physicians in case of the presence.

\section{Statistical analysis}

Data analysis was performed using SPSS 20 statistical software. The qualitative variables were described using frequency and percentages, and quantitative variables were described using range, mean, and standard deviation. Chi-square test was used to compare qualitative variables between the two groups and women's satisfaction with the methods; independent t-test was also used to compare the quantitative variables and the severity of pain between the two groups. $\mathrm{P}$ value $<0.05$ was considered as the significance level.

\section{Results}

Table (1): Comparison of personal characteristics between the lidocaine infiltration group I and LP cream group II.

\begin{tabular}{|c|c|c|c|c|c|}
\hline \multirow{3}{*}{ Variables } & \multicolumn{4}{|c|}{ Group } & \multirow[b]{3}{*}{ P. value } \\
\hline & \multicolumn{2}{|c|}{ Lidocaine infiltration $(n=72)$} & \multicolumn{2}{|c|}{ LP cream $(n=72)$} & \\
\hline & No. & $\%$ & No. & $\%$ & \\
\hline \multicolumn{6}{|l|}{ Age } \\
\hline Mean \pm SD & \multicolumn{2}{|c|}{$26.22 \pm 3.96$} & \multicolumn{2}{|c|}{$27.08 \pm 5.85$} & 0.303 \\
\hline \multicolumn{6}{|l|}{ Residence } \\
\hline Rural & 65 & 90.3 & 63 & 87.5 & \multirow[t]{2}{*}{0.596} \\
\hline Urban & 7 & 9.7 & 9 & 12.5 & \\
\hline \multicolumn{6}{|l|}{ Educational level } \\
\hline Illiterate & 21 & 29.2 & 29 & 40.3 & \multirow{5}{*}{0.176} \\
\hline Read and write & 25 & 34.7 & 14 & 19.4 & \\
\hline Preparatory & 6 & 8.3 & 6 & 8.3 & \\
\hline Secondary & 13 & 18.1 & 19 & 26.4 & \\
\hline University & 7 & 9.7 & 4 & 5.6 & \\
\hline \multicolumn{6}{|l|}{ Occupation } \\
\hline Employed & 5 & 6.9 & 4 & 5.6 & \multirow[b]{2}{*}{0.731} \\
\hline House wife & 67 & 93.1 & 68 & 94.4 & \\
\hline
\end{tabular}

(P: Significant Value) 
Table (2): Comparison of current pregnancy and labour outcomes between the lidocaine infiltration group I and LP cream group II.

\begin{tabular}{|c|c|c|c|c|c|}
\hline \multirow{3}{*}{ Variables } & \multicolumn{4}{|c|}{ Group } & \multirow{3}{*}{ P. value } \\
\hline & \multicolumn{2}{|c|}{$\begin{array}{l}\text { Lidocaine infiltration } \\
(n=72)\end{array}$} & \multicolumn{2}{|r|}{$\begin{array}{c}\text { LP cream } \\
(n=72)\end{array}$} & \\
\hline & No. & $\%$ & No. & $\%$ & \\
\hline \multicolumn{6}{|l|}{ Parity } \\
\hline Primipara & 1 & 1.4 & 7 & 9.7 & \multirow[b]{2}{*}{$0.029 *$} \\
\hline Multipara & 71 & 98.6 & 65 & 90.3 & \\
\hline \multicolumn{6}{|c|}{ Gestational age } \\
\hline Mean \pm SD & \multicolumn{2}{|c|}{$38.56 \pm 1.16$} & \multicolumn{2}{|r|}{$38.90 \pm 1.15$} & 0.074 \\
\hline $37-40$ & 70 & 97.2 & 66 & 91.7 & \multirow[b]{2}{*}{0.146} \\
\hline$>40$ & 2 & 2.8 & 6 & 8.3 & \\
\hline \multicolumn{6}{|c|}{ Onset of labour } \\
\hline Spontaneous & 57 & 79.2 & 50 & 69.4 & \multirow[b]{2}{*}{0.182} \\
\hline Augmented & 15 & 20.8 & 22 & 30.6 & \\
\hline \multicolumn{6}{|c|}{ Type of augmentation } \\
\hline Oxytocin & 9 & 60.0 & 19 & 86.4 & \multirow[t]{2}{*}{0.066} \\
\hline R.O.M & 6 & 40.0 & 3 & 13.6 & \\
\hline \multicolumn{6}{|c|}{ Birth weight (gm.) } \\
\hline Mean \pm SD & \multicolumn{2}{|c|}{$3200 \pm 473.82$} & \multicolumn{2}{|r|}{$3183.33 \pm 465.12$} & 0.832 \\
\hline$<2500$ & 7 & 9.7 & 8 & 11.1 & \multirow[b]{3}{*}{0.525} \\
\hline $2500-3500$ & 59 & 81.9 & 54 & 75.0 & \\
\hline 4000 \&more & 6 & 8.3 & 10 & 13.9 & \\
\hline \multicolumn{6}{|c|}{ Head circumference $(\mathrm{cm})$} \\
\hline Mean \pm SD & \multicolumn{2}{|c|}{$34.31 \pm 1$} & \multicolumn{2}{|r|}{$34.44 \pm 1.11$} & 0.455 \\
\hline
\end{tabular}

Table (3): Comparison of perineal tear \& its repair characteristics between lidocaine infiltrations group I and LP cream group II

\begin{tabular}{|c|c|c|c|c|c|}
\hline \multirow{3}{*}{ Variables } & \multicolumn{4}{|c|}{ Group } & \multirow{3}{*}{ P. value } \\
\hline & \multicolumn{2}{|c|}{$\begin{array}{c}\begin{array}{c}\text { Lidocaine infiltration } \\
(\mathrm{n}=72)\end{array} \\
\end{array}$} & \multicolumn{2}{|r|}{$\begin{array}{c}\text { LP cream } \\
(n=72)\end{array}$} & \\
\hline & No. & $\%$ & No. & $\%$ & \\
\hline \multicolumn{6}{|c|}{ Degree of perineal tear } \\
\hline $1^{\text {st }}$ degree & 8 & 11.1 & 28 & 38.9 & \multirow[b]{3}{*}{$0.001 *$} \\
\hline $2^{\text {nd }}$ degree & 63 & 87.5 & 41 & 56.9 & \\
\hline $3^{\text {rd }}$ degree & 1 & 1.4 & 3 & 4.2 & \\
\hline \multicolumn{6}{|c|}{ Duration of perineal repair (min) } \\
\hline Mean \pm SD & & .75 & & $6.37 \pm 3.68$ & $0.001 *$ \\
\hline \multicolumn{6}{|c|}{ Additional need of anesthesia } \\
\hline Yes & 10 & 13.9 & 14 & 19.4 & \multirow[b]{2}{*}{0.371} \\
\hline No & 62 & 86.1 & 58 & 80.6 & \\
\hline \multicolumn{6}{|c|}{ Side effects of anesthetics used } \\
\hline Tingling & 0 & 0.0 & 2 & 2.8 & \multirow[b]{4}{*}{0.171} \\
\hline Swelling & 2 & 2.8 & 0 & 0.0 & \\
\hline $\begin{array}{l}\text { Burning } \\
\text { Sensation }\end{array}$ & 0 & 0.0 & 1 & 1.40 & \\
\hline Non & 70 & 97.2 & 69 & 95.8 & \\
\hline
\end{tabular}




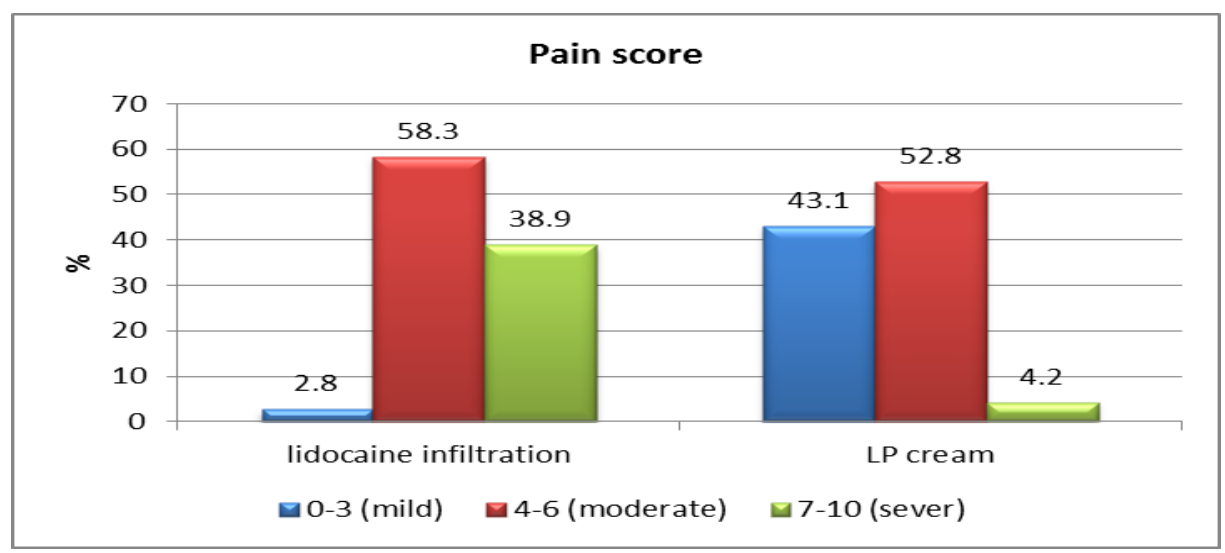

Fig. (1): Comparison between lidocaine infiltration \& LP cream group according to pain score. This figure shows statistical significant difference $(p=0.001)$ between both groups.

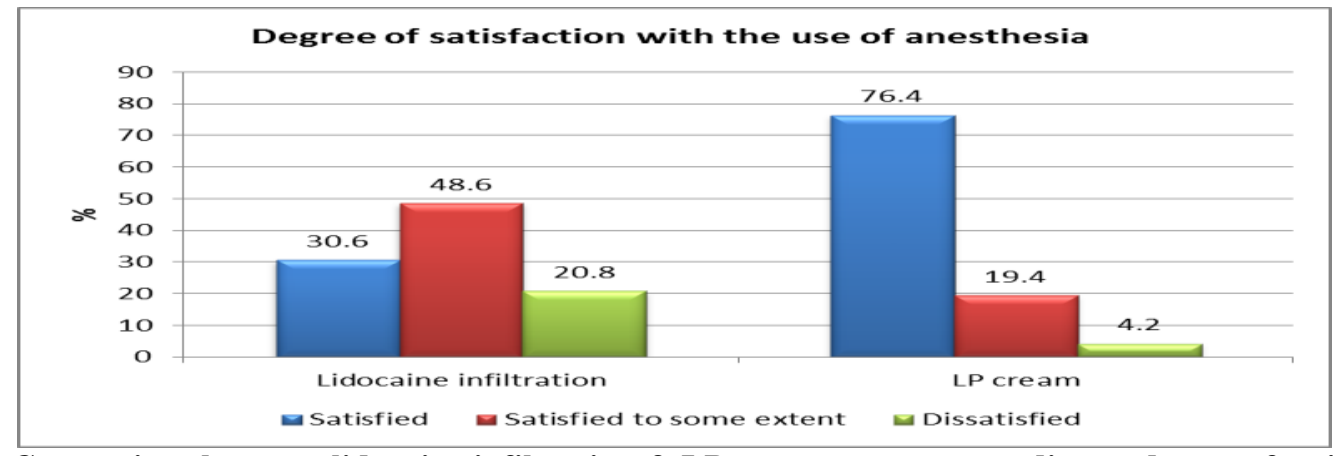

Fig. (2) Comparison between lidocaine infiltration \& LP cream groups according to degree of satisfaction with the use of anesthesia

This figure shows statistical significant difference $(p=0.001)$ between both groups.

No statistical difference as regard age, residence, educational level and occupation between lidocaine infiltration group I and LP cream group II where $(\mathrm{P}=$ $0.303,0.596,0.176 \& 0.731)$ respectively Table (1): The study includes 1 primipara in group I versus 7 in group II. The mean \pm SD gestational age is $38.56 \pm$ 1.16 in lidocaine group and $38.90 \pm 1.15$ in group II. $79.2 \%$ of labour occurs spontaneously in group I versus $69.4 \%$ in LP group Table (2): The vast majority of women $(87.5 \%)$ in lidocaine group and more than half of them $(56.9 \%)$ in LP group have $2^{\text {nd }}$ degree tear while $1^{\text {st }}$ and $3^{\text {rd }}$ degree represent $(11.1 \%$ $\& 1.4 \%)$ and $(38.9 \% \& 4.2 \%)$ respectively in both groups; the mean $\pm \mathrm{SD}$ duration of perineal repair is $(8.17 \pm 2.75 \& 6.37 \pm 3.68)$ respectively in both groups $(\mathrm{P}=0.001)$. No significant difference in the side effects and additional need of anesthetics used $(\mathrm{P}=0.171,0.371)$ in both groups respectively Table (3): In addition there is statistical significant difference $(p=0.001)$ between the two groups regarding pain scores Figure (1): and about three fourths $(76.4 \%)$ of women in LP group are satisfied compared to $(30.6 \%)$ in the lidocaine group $(\mathrm{p}=$ 0.001) Figure (2).

\section{Discussion}

Women have expressed pain related to perineal trauma in the postnatal period but little is known about pain during suturing. In addition, there is a lack of evidence to identify how professional decisions are made about pain management during the suturing process Despite, effective pain management is important in terms of both physical and psychological outcomes and has the potential to enhance the women's overall experience (Briscoe et al., 2015). Topical anesthetic creams may in some cases replace injected local anesthetics. They are much more "patient-friendly", as they eliminate needle fear and pain in the beginning of the procedure. It has been shown that by using a topical anesthetic cream the patient's anxiety and distress of the procedure itself are alleviated (Gyftopoulos, 2012).

The current study has assessed perineal pain level and overall mother's satisfaction by comparing the analgesic effect of topical application of lidocaineprilocaine cream and lidocaine infiltration during repair of postnatal spontaneous perineal tears.

Concerning the pain score, which was the main outcome, the present study found statistical 
difference between the lidocaine and the LP group $(\mathrm{p}=0.001)$ in which the mean \pm SD pain score in the lidocaine group was higher than mean \pm SD pain score in the LP group. The study also found that moderate pain scores was more than half and the severe pain was two fifth in the lidocaine group compared to two fifth of mild pain, slightly more than half of moderate pain with only $4.2 \%$ of severe pain in the LP group. Based on the study conducted via the same method by Franchi et al., (2009); who carried out a randomized controlled trial conducted at a referring academic institution, Italy; compared the effect of applied lidocaine-prilocaine cream to that of the local anaesthetics mepivacaine $1 \%$ infiltration in 61 women for providing pain relief during perineal suturing women in the EMLA group had lower pain score than those in the mepivacaine group $(\mathrm{P}=0002)$. This finding was in contrast with Duhan \& Nandal, (2013) who investigated the effect of the topical route administration of the lidocaine-prilocaine (EMLA) cream in comparison to the conventional perineal infiltration of lignocaine for suturing episiotomy. This study carried out in labour ward in the Department of Obstetrics \& Gynecology of a tertiary care center of North India on 100 primigravid women were randomly allocated into two groups. Group 1(50 women) received $10 \mathrm{ml}$ of $1 \%$ lignocaine for perineal infiltration at the time of crowning while the other 50 women of group 2 had EMLA cream application on the perineum at $8-9 \mathrm{~cm}$ of cervical dilatation during labor. In this study the mean \pm SD pain score was comparable in the lignocaine and the EMLA group $(p=0.487)$ and the vast majority of women in each group had a pain score of 6 or lower (mild to moderate pain). The cause of difference between the current study and Duhan et al. study might returned to that the study of Duhan et al. conducted only on primiparous which might have pain perception higher than multiparous women and also might be due to change in sample size between the studies in which the sample were 144 women in the present study vs 100 women in Duhan et al. study. Also the current result was not in the line with Kargar et al., (2016) whose study compared the effects of lidocaine prilocaine (EMLA) cream and lidocaine injection on reduction of perineal pain during perineal repair in normal vaginal delivery at Tehran, Iran in which 46 primiparous women were selected and randomly divided into two groups; EMLA and lidocaine group. This study mentioned that there was no difference between both groups (lidocaine and EMLA group) in the term of the pain score $(\mathrm{p}=0.730)$. The difference between the current study and Kargar et al study in this point has returned to the very small sample size (43 women) used by Kargar et al and difference in pain perception between women of different countries.

Regarding the degree of satisfaction with the use of anaesthesia, the current study demonstrated that one third of women in the lidocaine group expressed satisfaction, less than half were satisfied to some extent and only one fifth of them expressed dissatisfaction compared to three quarters in the LP group expressed satisfaction, one fifth were satisfied to some extent and only $4.2 \%$ expressed dissatisfaction with statistical difference between both groups $(\mathrm{p}=0.001)$; in which three quarters of women were satisfied in LP group vs one third in the lidocaine group. This result agreed with Duhan \& Nandal, (2013) This study carried out in labour ward in the Department of Obstetrics \& Gynecology of a tertiary care center of North India; who concluded that three quarters of women in lignocaine group and the vast majority of them in EMLA group were satisfied with the presence of statistical difference between the two groups $(p=0.04)$. Also the current finding matched the finding of Franchi et al., (2009) who carried out a randomized controlled trial conducted at a referring academic institution, Italy; where the majority of women expressed satisfaction with anesthesia method in the EMLA group, compared to slightly more than half in the mepivacaine group $(\mathrm{P}=0.01)$. But the current study in the term of satisfaction with anaesthesia used disagreed with Kargar et al., (2016) whose study compared the effects of lidocaine prilocaine (EMLA) cream and lidocaine injection on reduction of perineal pain during perineal repair in normal vaginal delivery at Tehran, Iran; found no difference between the two groups in degree of satisfaction $(p=0.681)$ where there was two fifth of women in lidocaine group vs one third in EMLA group fully satisfied and slightly more than half in lidocaine group were partial satisfied compared to two third in EMLA group. The causes of this difference between the two studies not major differences as the Kargar et al.'s study indicated that EMLA cream is as effective as lidocaine injection and makes the similar level of satisfaction and the difference might be due to difference in sample size where Kargar et al. used a very small sample size 43 women compared to 144 women recruited in the present study, also some changes in inclusion criteria might be another reason where Kargar et al. included only primiparous women, which may affect their perception than multiparous women and individual differences may be another cause. 


\section{Conclusions}

The findings of this trial concluded that

- Pain and satisfaction are better when using LP cream than using lidocaine infiltration during repair of postnatal spontaneous perineal tears.

- Topical anaesthetics appears to be safe, has no serious reactions, better tolerated, as it reduces painful infiltration, needle anxiety and complications of accidental injection of local infiltration.

- Topical anaesthetics are more easily to use compared to local injection.

\section{Recommendations}

- Train nurses regarding avoidance of perineal tear, different methods of anesthetic agent used during perineal repair.

- As the pain is the most common problem in the postnatal period, we recommend emphasizing the importance of postnatal care and good pain management by the nurse.

\section{References}

1. Bick Debra E., Ismail Khaled M., Macdonald Sue, Thomas Peter, Tohill Sue, \& Kettle Christine. (2013): How good are we at implementing evidence to support the management of birth related perineal trauma? A uk wide survey of midwifery practice. BMC Pregnancy and Childbirth, 12(1), 1-10.

2. Briscoe Lesley, Lavender Tina, O'Brien Ediri, Campbell Malcom, \& McGowan Linda. (2015): A mixed methods study to explore women and clinician's response to pain associated with suturing second degree perineal tears and episiotomies [praise]. Midwifery, 31(4), 464-72.

3. Dönmez Sevgul, \& Kavlak Oya. (2015): Effects of prenatal perineal massage and kegel exercises on the integrity of postnatal perine. Health, 07(04), 495.

4. Duhan Nirmala, \& Nandal Ritu. (2013): Topical lidocaine- prilocaine cream versus lignocaine infiltration for episiotomy repair: A randomized clinical trial. Journal of Clinical Research \& Governance, 2(2).

5. East Christine E., Sherburn Margaret, Nagle Catherine, Said Joanne, \& Forster Della. (2012): Perineal pain following childbirth: Prevalence, effects on postnatal recovery and analgesia usage. Midwifery, 28(1), 93-7.

6. Eshkevari Ladan, Trout Kimberly K., \& Damore Jennifer. (2013): Management of postpartum pain. Journal of Midwifery \& Women's Health, 58(6), 622-31.
7. Franchi M., Cromi A., Scarperi S., Gaudino F., Siesto G., \& Ghezzi F., (2009): Comparison between lidocaine-prilocaine cream (emla) and mepivacaine infiltration for pain relief during perineal repair after childbirth: A randomized trial. Am J Obstet Gynecol, 201(2), 186 e1-5.

8. Gyftopoulos K., (2012). The use of topical cream anesthetics in office procedures of the external genitalia Clinical use of local anesthetics (1st ed., pp. 102): INTECH open.

9. Kargar R., Aghazadeh-Nainie A., \& Khoddami-Vishteh H., (2016): Comparison of the effects of lidocaine prilocaine cream (emla) and lidocaine injection on reduction of perineal pain during perineum repair in normal vaginal delivery. J Family Reprod Health, 10(1), 21-6.

10. Laine Katariina, Räisänen Sari, \& Kalis Vladimir. (2016): Perineal trauma at childbirth. springer, 83-96.

11. Manzanares Sebastian, Cobo Davinia, Moreno-Martínez Maria, Sánchez-Gila Mar, \& Pineda Alicia. (2013): Risk of episiotomy and perineal lacerations recurring after first delivery. Birth, 40(4), 307-11.

12. Moore Emma, \& Moorhead Carolyn. (2013): Promoting normality in the management of the perineum during the second stage of labour. British Journal of Midwifery, 21(9), 616-20. 